nephron

Practice
Nephron 2019;143:207-210

DOI: $10.1159 / 000500421$
Received: March 26, 2019

Accepted after revision: April 13, 2019

Published online: May 7, 2019

\title{
Targeting Acute Kidney Injury: Can an Innovative Approach to Existing and Novel Biomarkers Shift the Paradigm?
}

\author{
Rajit K. Basu \\ Children's Healthcare of Atlanta, Division of Critical Care, Department of Pediatrics, Emory School of \\ Medicine, Atlanta, GA, USA
}

\section{Keywords}

Acute kidney injury · Intensive care $\cdot$ Biomarkers

\begin{abstract}
Acute kidney injury (AKI) continues to be an epidemic problem among hospitalized patients. Diagnostic stasis has, in part, contributed to the poor outcomes associated with AKI. Incorporation of newer diagnostic methodology, longitudinal assessment of markers of AKI alone and in combination may improve appreciation of patient risk and the speed of recognition, augment the precision of diagnosis, and target management.

(c) 2019 S. Karger AG, Basel
\end{abstract}

Acute kidney injury (AKI) continues to be a problem of epidemic proportions. Across age ranges, illness severity, and geographic distribution, hospitalized patients with AKI are subject to worse outcomes compared to those without AKI: increased hospital resource utilization (mechanical ventilation, length of stay), higher costs of care, and increased rate of death $[1,2]$. Given the increasing broader recognition of both AKI and associated complications, a contemporary and renewed approach to the injury syndrome is warranted.

Multiple factors drive the association between AKI and downstream outcome(s). The lack of therapeutic options and concomitant emphasis on supportive manage-

\section{KARGER}

() 2019 S. Karger AG, Basel

E-Mail karger@karger.com

www.karger.com/nef ment, an inherently reactive strategy, is secondary in part to limited diagnostic tools used in practice. The alignment of AKI recognition has been made facile by the adoption of stratification systems such as RIFLE, AKIN, and KDIGO. Diagnosis and monitoring of AKI, however, has largely been dependent on one determinant (change in serum creatinine [SCr] from baseline and then from day to day). The importance of urine output assessment has only recently been highlighted $[3,4]$. These markers carry known limitations - including only affording the ability to identify AKI broadly and without precision to type of injury. The management of critically ill patients, however, is improved by specificity for the type of injury, as these patients are constantly changing with regards to individual organ stability and function and also overall homeostatic balance. Respiratory failure is an apt example. Progressive respiratory distress is assessed, managed, and treated in a sequential, iterative way. Multiple diagnostic inputs ranging from physical exam, radiography, blood gas assessment, capnography, to pulse oximetry are used over time (and in the context of global stability) to

Contribution from the AKI and CRRT 2019 Symposium at the 24th International Conference on Advances in Critical Care Nephrology, Manchester Grand Hyatt, San Diego, CA, USA, February 26 - March 1, 2019. This symposium was supported in part by the NIDDK funded University of Alabama at Birmingham-University of California San Diego O'Brien Center for Acute Kidney Injury Research (P30DK079337).
Rajit K. Basu, MD, MS, FCCM

Children's Healthcare of Atlanta, Division of Critical Care Department of Pediatrics, Emory School of Medicine Atlanta, GA 30322 (USA)

E-Mail rajit.basu@choa.org 
personalize the approach to a patient. Interventions have concurrently evolved over time, spanning the breadth of specific support for impairments in oxygenation, ventilation, secretion load, bronchospasm, and so on. A dynamic diagnostic approach to AKI, mirroring the approach to respiratory failure, to integrate multiple inputs along the trajectories of critically ill patients, may ultimately lead to more precise and effective therapeutic options.

A precise understanding of what drives AKI is incomplete. Problematically, the concordance of SCr change with renal injury, the mainstay of alerting a provider to the presence or development of kidney insult, is fraught with limitation. The multiple weaknesses of using $\mathrm{SCr}$ for diagnosis have been highlighted repeatedly but are particularly relevant in younger and smaller patients. The ubiquitous prevalence of the test, moreover, has propagated the misclassification of $\mathrm{SCr}$ changes into broad, outdated and imprecise umbrella categories of "pre-renal" or "intrinsic" AKI. For example, the nomenclature "pre-renal" theoretically defines, in one term, severity, timing, duration, and reversibility of injury, while simultaneously underscoring the recommended therapy (i.e., fluid administration). A patient with congestive heart failure, however, fulfills diagnostic criterion for pre-renal AKI but violates the aforementioned descriptions of injury and could be significantly harmed by such a one-size-fits-all approach. Further, the pathobiology of pre-renal AKI, often attributed to volume-depletion or ischemic AKI, demonstrates marked heterogeneity in acute gene dysregulation and adaptive or maladaptive protein expression in the kidney [5]. There is also limited histologic evidence supporting the dogma equating intrinsic AKI to acute tubular necrosis.

The existing definition of AKI is being challenged. The Acute Dialysis and Quality Initiative (ADQI) international consensus panels have been instrumental in shifting the current paradigm. The 10th ADQI advocated changes to improve the precision of AKI nomenclature, moving away from terms such as pre-renal and intrinsic renal to more pathophysiologic terms such functional or tubular damage-associated AKI [6]. In addition to the delineation of how time course relates to functional AKI, persistent AKI, and acute kidney disease, the 16th ADQI recommended a study (including risk scores, functional markers, and use of biomarkers) to identify, predict, and further characterize patients with persistent AKI (i.e., $\geq 48 \mathrm{~h}$ of SCr elevation or oliguria) [7]. The renal angina prodrome of AKI risk stratification, attempts to integrate both of these ADQI objectives. Incorporating early signs of kidney injury with patient context into the renal angina index identifies the patients with the highest pre-test probability (risk) for evolution into severe AKI after $72 \mathrm{~h}$ [8]. More evidence now attempts to improve the delineation of the proteomics and biology of disease, including hemodynamic and bioenergetic drivers of AKI [9]. Understanding of the kidney's response to insult, as assessed by the adaptive or maladaptive responses to injury, is now being studied [9]. A preponderance of evidence indicates that excessive positive fluid balance (fluid overload) is associated with poor outcome, is simultaneously a sign of and a risk factor for AKI, and can mask AKI diagnosis $[10,11]$. Finally, a growing appreciation for the overlap of AKI with other organ dysfunction has led to an evolution in the understanding of cross-over syndromes of AKI (e.g., hepato-, pulmonary-, and cardio-). In total, the set of assumptions related to AKI - that is, understanding of the who, what, when, why, and how of injury has been challenged. The reframed understanding is more personalized, precise, and contemporary.

A contemporary, dynamic approach to diagnostics opens up possibilities to create a more targeted approach to AKI management. A combination of markers may delineate a more precise AKI phenotype. A combination approach can have multiple meanings: (1) the same marker, more than once (2) multiple markers at the same time, or (3) multiple markers more than once. A number of universally available options currently exist for use in a combination approach to AKI management. Creatinine change over time, assessed from one point to the next, can be integrated into the kinetic change in glomerular filtration rate [12]. The kinetic change in glomerular filtration rate significantly improves the identity the patients with AKI progression versus resolution (compared to looking at creatinine as a static variable). The incorporation of urine output assessment, along with SCr changes, increases the precision for AKI severity, in both adults and children [4]. The change in fluid balance over time is associated with patient outcome - implicating the importance of net fluid balance and associated markers (e.g., fluid overload, urine output by time) as "combination markers" with clinical importance for prognostication [13]. Adjudicating the concentration of SCr for net fluid balance (combining the markers of fluid and creatinine) refines the severity of AKI and associated downstream sequelae [11]. Objectively quantified fluid overload, in the context of SCr changes, can provide more information about AKI severity. Importantly, fluid balance is a modifiable parameter of critical illness and is adjudicated multiple times daily on patients. Understanding of the adaptive and maladaptive responses, highlighted earlier, may be further augmented by the adjudication of renal 


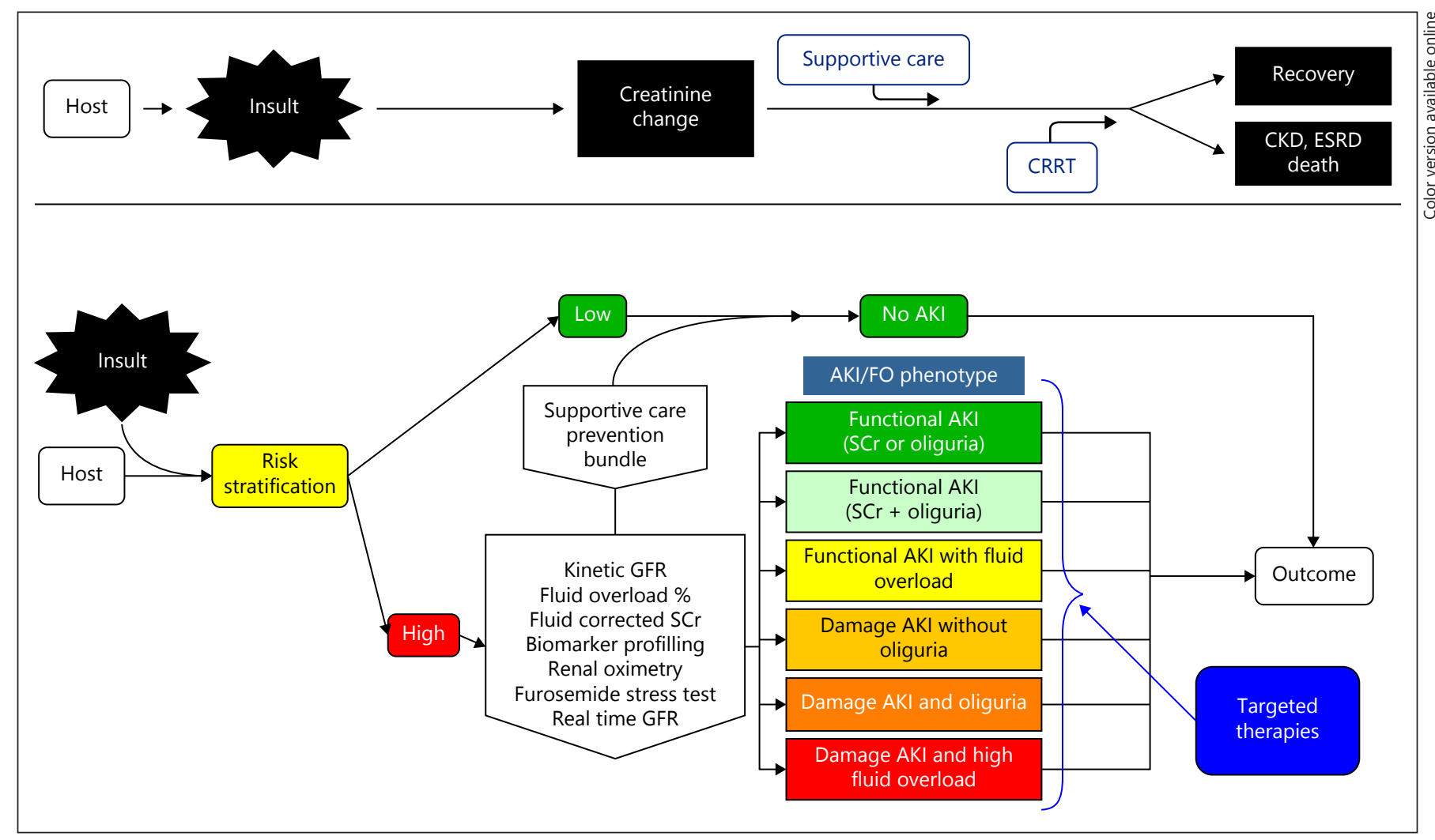

Fig. 1. The existing static and limited diagnostic strategy with the existing management options and associated epidemiology is juxtaposed with the potential paradigm change offered via a multimodal and longitudinal approach to AKI assessment, management, and therapeutics. Adjudication of AKI by different modalities, incorporating creatinine change ( $\mathrm{SCr}$ ) and UOP changes may identify specific phenotypes of AKI amenable to targeted therapy. RST, renal support therapy; CKD, chronic kidney disease; ESRD, end-stage renal disease; GFR, glomerular filtration rate; NAc, Nacetylcysteine; AKI, acute kidney injury; SCr, serum creatinine; UOP, urine output. reserve, as estimated by the response to a standardized dose of furosemide. The furosemide stress test, in combination with tubular markers, can identify with high precision, patients with tubular damage-associated severe AKI and those who will progress to receiving continuous renal replacement therapy [14]. Newer tubular damage markers most extensively studied, urinary neutrophil gelatinase associated lipocalin and tissue inhibitor of metalloproteinase-2/insulin-like growth factor binding protein 7, offer the opportunity to separate different severity phenotypes of AKI based on functional injury, renal "stress," or tubular damage. Combining functional and damage biomarkers identifies patients with creatinine elevation with functional or reversible injury with high predictive specificity compared to creatinine alone [15]. Additionally, the phenotype of "subclinical" AKI can be identified - patients without AKI by creatinine elevation on initial assessment and who are eventually affected by AKI and/or similar poor ICU and renal outcomes (length of stay prolongation, increased requirement of renal replacement therapy, higher rate of death).

Initial results suggest that targeting therapy based on innovative approaches to biomarker integration can modify the course of AKI. Randomization of patients based on biomarker elevation following cardiac surgery to an AKI management bundle or standard of care demonstrated a significant reduction in AKI severity and AKI duration [16]. Similar findings were demonstrated in general surgery patients.

In summary, critically ill patients affected by the AKI epidemic need a modern and personalized approach to care. Use of a conventional and relatively monocular diagnostic approach to AKI will likely perpetuate the poor outcomes associated with AKI. The potential exists to improve the understanding, diagnostics, and management of AKI with sophistication and precision (Fig. 1). 
Moving toward a dynamic and multimodal approach to AKI, paralleling the approach used for other critical illnesses, will reframe the paradigm and open the door to newer and targeted therapeutic possibilities in the future.

\section{Disclosure Statement}

Dr. Basu serves as a consultant for BioPorto Diagnostics and is on the Baxter Acute Therapies Institute Speakers Bureau.

\section{References}

1 Rewa O, Bagshaw SM. Acute kidney injuryepidemiology, outcomes and economics. Nat Rev Nephrol. 2014 Apr;10(4):193-207.

2 Kaddourah A, Basu RK, Bagshaw SM, Goldstein SL; AWARE Investigators. Epidemiology of Acute Kidney Injury in Critically Ill Children and Young Adults. N Engl J Med. 2017 Jan;376(1):11-20.

3 Kellum JA, Sileanu FE, Murugan R, Lucko N, Shaw AD, Clermont G. Classifying AKI by Urine Output versus Serum Creatinine Level. J Am Soc Nephrol. 2015 Sep;26(9):2231-8.

4 Kaddourah A, Basu RK, Goldstein SL, Sutherland SM; Assessment of Worldwide Acute Kidney Injury, Renal Angina and, Epidemiology (AWARE) Investigators. Oliguria and Acute Kidney Injury in Critically Ill Children: Implications for Diagnosis and Outcomes. Pediatr Crit Care Med. 2019 Apr;20(4):332-9.

5 Devarajan P. Acute kidney injury: Acute kidney injury: still misunderstood and misdiagnosed. Nat Rev Nephrol. 2017 Mar;13(3): $137-8$.

6 Endre ZH, Kellum JA, Di Somma S, Doi K, Goldstein SL, Koyner JL, et al. Differential diagnosis of AKI in clinical practice by functional and damage biomarkers: workgroup statements from the tenth Acute Dialysis Quality Initiative Consensus Conference. Contrib Nephrol. 2013;182:30-44.
7 Chawla LS, Bellomo R, Bihorac A, Goldstein SL, Siew ED, Bagshaw SM, et al.; Acute Disease Quality Initiative Workgroup 16. Acute kidney disease and renal recovery: consensus report of the Acute Disease Quality Initiative (ADQI) 16 Workgroup. Nat Rev Nephrol. 2017 Apr;13(4):241-57.

8 Basu RK, Zappitelli M, Brunner L, Wang Y, Wong HR, Chawla LS, et al. Derivation and validation of the renal angina index to improve the prediction of acute kidney injury in critically ill children. Kidney Int. 2014 Mar; 85(3):659-67.

9 Okusa MD, Rosner MH, Kellum JA, Ronco C Acute Dialysis Quality Initiative XIII Workgroup. Therapeutic Targets of Human AKI: Harmonizing Human and Animal AKI. J Am Soc Nephrol. 2016 Jan;27(1):44-8.

10 Alobaidi R, Morgan C, Basu RK, Stenson E, Featherstone R, Majumdar SR, et al. Associations Between Fluid Balance and Outcomes in Critically Ill Children: A Protocol for a Systematic Review and Meta-analysis. Can J KidneyHealthDis.2017Feb;4:2054358117692560.

11 Macedo E, Bouchard J, Soroko SH, Chertow GM, Himmelfarb J, Ikizler TA, et al.; Program to Improve Care in Acute Renal Disease Study. Fluid accumulation, recognition and staging of acute kidney injury in critically-ill patients. Crit Care. 2010;14(3):R82.
12 Chen S: Kinetic Glomerular Filtration Rate Equation Can Accommodate a Changing Body Volume: Derivation and Usage of the Formula. Math Biosci 2018 Dec;306:97-106.

13 Silversides JA, Fitzgerald E, Manickavasagam US, Lapinsky SE, Nisenbaum R, Hemmings $\mathrm{N}$, et al.; Role of Active Deresuscitation After Resuscitation (RADAR) Investigators. Deresuscitation of Patients With Iatrogenic Fluid Overload Is Associated With Reduced Mortality in Critical Illness. Crit Care Med. 2018 Oct;46(10):1600-7.

14 Koyner JL, Davison DL, Brasha-Mitchell E, Chalikonda DM, Arthur JM, Shaw AD, et al. Furosemide Stress Test and Biomarkers for the Prediction of AKI Severity. J Am Soc Nephrol. 2015 Aug;26(8):2023-31.

15 Basu RK, Wong HR, Krawczeski CD, Wheeler DS, Manning PB, Chawla LS, et al. Combining functional and tubular damage biomarkers improves diagnostic precision for acute kidney injury after cardiac surgery. J Am Coll Cardiol. 2014 Dec;64(25):2753-62.

16 Meersch M, Schmidt C, Hoffmeier A, Van Aken $\mathrm{H}$, Wempe C, Gerss J, et al. Prevention of cardiac surgery-associated AKI by implementing the KDIGO guidelines in high risk patients identified by biomarkers: the PrevAKI randomized controlled trial. Intensive Care Med. 2017 Nov;43(11):1551-61. 